\title{
Posições verticalizadas no parto e a prevenção de lacerações perineais: revisão sistemática e metanálise*
}

\author{
Upright positions in childbirth and the prevention of perineal lacerations: \\ a systematic review and meta-analysis \\ Posiciones verticales en el parto y prevención de laceraciones perineales: \\ revisión sistemática y metaanálisis
}

Como citar este artigo:

Rocha BD, Zamberlan C, Pivetta HMF, Santos BZ, Antunes BS. Upright positions in childbirth and the prevention of perineal lacerations: a systematic review and meta-analysis. Rev Esc Enferm USP. 2020;54:e03610. doi: https://doi.org/10.1590/S1980-220X2018027503610

Bruna Dedavid da Rocha ${ }^{1}$

Cláudia Zamberlan ${ }^{1}$

Hedioneia Maria Foletto Pivetta ${ }^{2}$

Bianca Zimmermann Santos ${ }^{1}$

Bibiana Sales Antunes ${ }^{1}$

* Extraído da dissertação: "Posições verticalizadas no parto e a prevenção de lacerações perineais: revisão sistemática e metanálise", Universidade Franciscana, 2017

${ }^{1}$ Universidade Franciscana, Programa de Pós-Graduação em Saúde Materno Infantil, Santa Maria, RS, Brasil.

${ }^{2}$ Universidade Federal de Santa Maria, Departamento de Fisioterapia e Reabilitação, Santa Maria, RS, Brasil.

\begin{abstract}
Objective: To investigate whether the adoption of upright positions by women during childbirth prevents perineal lacerations compared to the lithotomy position. Method: A systematic review with meta-analysis. The searches were carried out in the databases: LILACS, Medline/PubMed, CINAHH, Cochrane Library, Web of Science, Science Direct and Scopus. Searches in the gray literature were conducted on Google Scholar and OpenGrey databases. Reference lists of included articles were also considered. The Cochrane collaboration tool and ACROBAT-NRSI were used to analyze the methodological quality of the articles. Results: There were 26 studies listed and 8 were selected for the meta-analysis. The level of scientific evidence was classified by the GRADE System and considered high. There was no statistically significant difference between upright positions in relation to horizontal positions. Despite this finding, the upright positions showed reduced rates of severe perineal lacerations. Conclusion: Adopting upright positions in normal delivery can be encouraged by professionals as it can prevent severe perineal lacerations; however, it is not possible to accurately affirm their effectiveness to the detriment of horizontal positions for an intact perineum outcome.
\end{abstract}

\section{DESCRIPTORS}

Obstretric Nursing; Parturition; Modalities, Position; Lacerations; Review; Meta-Analysis. 


\section{INTRODUÇÃO}

O desenvolvimento tecnológico, a interferência médica no cenário do parto, assim como, a hipermedicalização da prática obstétrica tornou-se rotina nas instituições de saúde. O Brasil é considerado o país com as mais elevadas taxas de cesarianas e de morbimortalidade materna e perinatal do mundo. Portanto, existe premência em fortalecer o sistema de saúde, para que seja proporcionada uma assistência humanizada, individualizada e de qualidade para todas as mulheres ${ }^{(1)}$.

De acordo com resultados da pesquisa nacional "Nascer no Brasil", o país possui altos índices de cesarianas e intervenções, com a justificativa de precipitar o processo de parto e nascimento. A indução do parto com ocitocina sintética, realização da Manobra de Kristeller, episiotomia e a permanência da mulher em posição litotômica, são algumas das práticas mais utilizadas e apresentadas pela pesquisa. Portanto, conclui-se que o processo de parto e nascimento é conduzido, preponderantemente, pelo profissional, de forma que a mulher, que deveria ser protagonista, torna-se coadjuvante desse processo $^{(2)}$.

A trajetória da saúde da mulher, no Brasil, está avançando consideravelmente, em termos de políticas públicas. Na perspectiva de um modelo qualificado e humanizado de assistência, que visa minimizar riscos maternos e neonatais, tem-se o Programa de Humanização do Parto e Nascimento $(\mathrm{PHPN})^{(3)}$. Destaca-se também a Política Nacional de Atenção Integral à Saúde da Mulher (PNAISM), pois, a partir desta, aspectos inerentes à saúde materno-infantil foram ampliados, como o estímulo ao empoderamento e protagonismo da mulher, para além da maternidade, incluindo as questões de gênero e considerando os direitos humanos, com o propósito de reduzir a morbimortalidade por causas preveníveis e evitáveis ${ }^{(4)}$. Em termos de gestão da atenção à saúde materno-infantil, a iniciativa Rede Cegonha foi implementada com o propósito de garantir acesso aos serviços de saúde, acolhimento e resolutividade na área obstétrica, por meio da melhoria da qualidade dos serviços e da capacitação profissional ${ }^{(5)}$.

No âmbito internacional, a Organização Mundial da Saúde (OMS) defende que, para uma boa condução do parto normal, os profissionais devem nortear sua prática nas melhores evidências científicas realizadas. A partir disso, defende que dentre as "Boas Práticas de Atenção ao Parto e Nascimento" destacam-se a liberdade de posição e movimentação durante o trabalho de parto, o estímulo às posições verticalizadas no parto e a prática restritiva da episiotomia. Esse documento atualizado em 2018, reforça a recomendação de que o profissional de saúde não deve impor uma posição de parto à mulher, mas sim estimular a livre escolha de posição, incluindo as verticais, priorizando o conforto e respeitando o desejo da mulher ${ }^{(6)}$.

De acordo com as melhores evidências científicas internacionais, a posição litotômica no momento do parto, aumenta a ocorrência de partos vaginais instrumentados, dor perineal e frequência cardíaca fetal não tranquilizadora. Portanto, as mulheres devem ser desencorajadas a adotarem a posição litotômica e encorajadas a escolher uma posição que lhes seja mais confor-

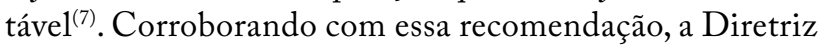
Nacional de Assistência ao Parto Normal ainda acrescenta que devem ser incluídas diferentes posições como cócoras, lateral e quatro apoios ${ }^{(8)}$.

Cabe ressaltar a relevância das evidências científicas para a qualificação da prática em saúde materno-infantil. Nesse enfoque, destaca-se a importância da implementação da prática baseada em evidências na enfermagem, visto que, diversas maternidades, centros de parto normal e hospitais do Brasil, estão incorporando resultados das sínteses de revisões sistemáticas e metanálises na sua prática. A partir disso, principalmente as enfermeiras obstétricas e obstetrizes, tem um papel fundamental na implementação das evidências científicas, na rotina desses serviços de saúde $\mathrm{e}^{(9)}$. Porém, ainda existem lacunas significativas de produções científicas na área, em se tratando da eficácia e aplicabilidade das boas práticas, aliadas ao conhecimento clínico, fato este que, se implementado, pode contribuir com o processo de gestão nas maternidades brasileiras, em especial, as de risco habitual.

No que se refere à incorporação das evidências científicas na prática obstétrica, a literatura sinaliza barreiras no processo de trabalho dos profissionais e na organização estrutural dos serviços de saúde, como a alta demanda de trabalho, precariedade da estrutura física e reduzido número de leitos e profissionais para atender o número de partos das instituições. Também salienta-se limitações relacionadas à formação dos profissionais, principalmente da área médica, ainda pautada no modelo biológico, anterior à políticas públicas de humanização, voltada para as intervenções no parto, o que pode ser observado pela resistência dos mesmos em aderir às boas práticas e romper o paradigma tecnicista de cuidado ${ }^{(9-10)}$.

Com base nessas considerações, e pautado no processo de organização das redes de atenção em saúde materno-infantil, aliado às evidências clínicas, este estudo objetivou realizar uma revisão sistemática de literatura com metanálise, sobre a adoção de posições verticalizadas pela mulher, no momento do parto, comparada à posição litotômica, na prevenção de lacerações perineais. Neste estudo serão consideradas posições verticalizadas todas as adotas espontaneamente pela paciente, as quais diferem da posição convencional de litotomia (deitada na cama de parto/ maca), tais como cócoras (com ou sem uso de banqueta, sustentada pelo acompanhante ou não), semi lateral, lateral, quatro apoios, em pé. Posições horizontais são consideradas a posição de litotomia, com ou sem o uso de perneiras de apoio e posição dorsal.

\section{MÉTODO}

\section{Desenho DO ESTUdO}

Consiste em uma revisão sistemática com metanálise, a qual seguiu o percurso metodológico para elaboração de revisões sistemáticas, preconizado pelas Diretrizes 
Metodológicas para a Elaboração de Revisões Sistemáticas, do Ministério da Saúde. A construção desse documento ministerial baseou-se, principalmente, em diretrizes internacionais como as elaboradas pela "The Cochrane Reviewer's Handbook"(11) e pelo grupo "The Australian National Health and Medical Research Council"(12). Além disso, este estudo também foi norteado pelo protocolo para redação de manuscritos de revisão sistemática, o qual denomina-se recomendação PRISMA (Principais Itens para Relatar Revisões sistemáticas e Metanálises) ${ }^{(13)}$, elaborado em reunião realizada pelo The PRISMA Group, o qual inclui autores de revisões, metodologistas, clínicos, editores e um consumidor, os quais revisaram e expandiram o antigo fluxograma utilizado, denominado QUORUM (Qualidade dos Relatos de Metanálises).

Foi elaborado um protocolo da revisão sistemática, baseado no projeto de pesquisa, o qual foi registrado na base PROSPERO (International Prospective Register of Systematic Reviews), e que pode ser consultado na referida base a partir do protocolo CRD42016046322.

A partir disso, foram obedecidos os sete passos para elaboração de revisões sistemáticas e elaborada a seguinte questão norteadora da pesquisa, a partir da estratégia PICO (População; Intervenção; Comparação; Outcome): "A adoção de posições verticalizadas pela parturiente, no momento do parto, comparada à posição litotômica, previne lacerações perineais?”.

\section{Coleta de dados}

Foram realizadas buscas em bases de dados Medline/ PubMed, Lilacs (Literatura Latino-Americana e do Caribe em Ciências da Saúde), CINAHL (Cumulative Index to Nursing E' Allied Health Literature), Scopus (Scopus Info Site), Cochrane Library, Science Direct e Web of Science, e duas bases de literatura não convencional, também denominadas de "literatura cinzenta": Google Scholar e OpenGrey, no período de dezembro de 2016. Os principais descritores, adotados na estratégia de busca, indexados nos Descritores em Ciências da Saúde (DECS) e no Mesh Database (Medical Subject Headings), foram: segunda fase de trabalho de parto, posição, lacerações, e seus termos correspondentes em inglês, second stage labor, perineum, posture e lacerations, os quais foram combinados por meio do operador booleano $A N D$. Foram utilizados filtros em determinadas bases, excluindo outros formatos de publicação (teses, dissertações, comentários e livros) (Quadro 1).

\section{Critérios de seleção}

Foram selecionados estudos realizados com mulheres em trabalho de parto ativo, submetidas ao parto vaginal, que tiveram lacerações perineais no período expulsivo ou mantiveram seu períneo íntegro após o parto.

Em relação aos critérios de elegibilidade dos estudos, foram incluídas publicações de estudos primários na íntegra, com ou sem resumo disponível, que contemplassem parturientes em trabalho de parto ativo, que adotaram posições verticalizadas ou posição litotômica na segunda fase de trabalho de parto e a prevenção ou não de lacerações perineais, ao adotar as posições verticalizadas ou a posição litotômica, independente da paridade e da idade gestacional, sem intervenções como analgesia de parto e indução de trabalho de parto com ocitocina sintética.

Quadro 1 - Estratégia de busca nas bases de dados elencadas.

\begin{tabular}{|c|c|}
\hline$\# 1$ & second stage labor [MeSH Terms*] \\
\hline$\# 2$ & posture [MeSH Terms*] \\
\hline$\# 3$ & lacerations [MeSH Terms*] \\
\hline$\# 4$ & perineum $[$ MeSH Terms*] \\
\hline$\# 5$ & segunda fase de trabalho de parto $\left[\mathrm{DeCS}^{\dagger}\right]$ \\
\hline \#6 & posição [palavra-chave] \\
\hline$\# 7$ & períneo $\left[\mathrm{DeCS}^{+}\right]$ \\
\hline$\# 8$ & lacerações [DeCS $\left.{ }^{\dagger}\right]$ \\
\hline \multicolumn{2}{|r|}{$\begin{array}{l}\text { Medline/Pubmed } \text { /Scopus }^{\S} / \text { Web of Science: \#1 AND \#2 } \\
\text { CINAHL }{ }^{* *} / \text { Cochrane Library: \#1 AND \#2 AND \#3 (Títulos CINAHL } L^{* *} \text { - pesquisa como keywords; Cochrane Library - Title, abtract, keywords } \\
\text { Science Direct: \#1 AND \#2 AND \#4 (all fields) } \\
\text { Open Grey: } \# 1 \\
\text { Google Scholar: \#1 AND \#2 AND \#3 AND \#4; \#5 AND \#6 AND \#7 AND \#8 } \\
\text { LILACS }{ }^{+1} \text { : \#5 AND \#6 } \\
\text { Filtros: excluídas dissertações, teses, comentários e livros. }\end{array}$} \\
\hline
\end{tabular}

* Medical Subject Headings Terms

+ Descritores em Ciências da Saúde

‡ Public Medline

$\S$ Scopus Info Site

** Cumulative Index to Nursing \& Allied Health Literature

${ }^{++}$Literatura Latino-Americana e do Caribe em Ciências da Saúde 
Foram excluídos artigos que não estivessem relacionados à questão de pesquisa, ao desenho metodológico (não foram elencadas revisões, reflexões críticas, teses, dissertações, capítulos de livro e manuais ministeriais), participantes, tipo de intervenção, tipo de comparação e desfecho, e ainda estudos secundários, tais como: artigos de revisão e opinião, editoriais, teses, dissertações e capítulos de livro. Não foi delimitado recorte temporal ou idioma das publicações. As referências bibliográficas identificadas por meio da estratégia de busca, e que constituíram a amostra final, foram gerenciadas pelo software Mendeley.

A pesquisa não teve uma amostra de artigos pré-definida, sendo que, por ser uma temática ainda insipiente na literatura, na sua especificidade, foram esgotadas todas as possibilidades de seleção de publicações em formato de artigo nas bases de dados elencadas, a partir das estratégias de pesquisa escolhidas.

Referente a extração dos dados, a mesma foi composta de duas etapas realizadas de maneira independente, por dois revisores. Inicialmente, foi realizado o teste de relevância I, pelo qual procedeu-se a leitura do título e resumo dos estudos, sendo que, artigos sem resumo seriam avaliados somente pelo título e sua coerência com a temática. A pesquisadora principal elaborou uma ficha clinica padronizada para coleta de dados nessa fase. A segunda etapa consistiu no teste de relevância II, por meio da leitura dos artigos na íntegra. $\mathrm{Na}$ segunda etapa, a obtenção dos dados descritivos e quantitativos, relacionados às caraterísticas dos estudos, foi operacionalizada por meio de formulário próprio, o qual continha as seguintes informações: título do artigo, autores, área de atuação, periódico, ano de publicação, local do estudo, país, tema, objetivo, pergunta de pesquisa, metodologia, população, número da amostra, preceitos éticos, testes estatísticos, coleta de dados, nível de evidência, força da recomendação, comparação entre posição litotômica e posições verticalizadas, prevalência de cada posição e resultados. Após a seleção dos estudos, foi realizada a busca manual de artigos, por referência cruzada. Foi contatado um especialista na área obstétrica para decidir as divergências entre os dois revisores referente ao teste de relevância II. O grau de concordância dos revisores foi estabelecido pela medida de Cohen $\mathrm{Kappa}^{(11-12)}$ e o índice alcançado foi de 0,261, considerado moderadamente satisfatório. O fluxograma da revisão sistemática pode ser visualizado na página seguinte, pela Figura 1.
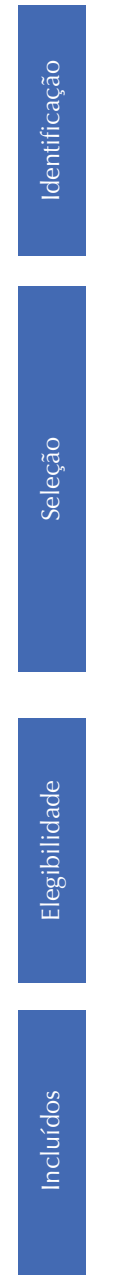

Registros identificados nas bases de dados $(n=1.341)$ 


\section{ANÁLISE E TRATAMENTO DOS DADOS}

Os dados foram analisados por meio de metanálise dos estudos clínicos randomizados (ECRs) e análise qualitativa de todos os estudos elencados. A qualidade metodológica dos estudos selecionados pelo teste de relevância II, foi avaliada por meio da Ferramenta da Colaboração Cochrane para Avaliação do Risco de Viés de Ensaios Clínicos Randomizados ${ }^{(14)}$ e também pela Ferramenta $A C R O B A T$ NRSI (A Cochrane Risk Of Bias Assessment Tool: for NonRandomized Studies of Interventions) $)^{(15)}$.

\section{Aspectos éticos}

Por se tratar de uma metodologia de pesquisa que não envolve a participação de seres humanos, não existe a necessidade de projetos de revisão serem avaliados por uma comissão de ética. Porém, devido à necessidade de contatar um(uma) especialista na temática para decidir divergências entre os dois revisores, após realização dos testes de relevância, o projeto foi enviado ao comitê de ética, conforme preconiza a Resolução no 466, de 12 de dezembro de 2012, do Conselho Nacional de Saúde, que trata de pesquisas envolvendo seres humanos. A partir disso, foi elaborado um Termo de Consentimento Livre e Esclarecido (TCLE) o qual foi assinado pelo(a) especialista. $O$ projeto de pesquisa recebeu parecer favorável do Conselho de Ética e Pesquisa, sob número 1.771.295, em 11 de outubro de 2016.

\section{RESULTADOS}

Foram utilizados dois métodos de avaliação, devido às diferentes metodologias dos estudos selecionados. $\mathrm{Na}$ avaliação pela ferramenta Cochrane, dos 13 estudos clínicos, a maioria foi classificado como baixo risco de viés, principalmente os que foram elencados para a metanálise. Pelo Acrobat, a maioria dos estudos de coorte e caso-controle também apresentaram baixo risco de viés. A qualidade da evidência foi avaliada pelas diretrizes do Sistema GRADE (Grading of Recommendations Assessment, Development and Evaluation) $)^{(16)}$.

Para a análise estatística, foi utilizado o software Review Manager 5.0, disponibilizado pela The Cochrane Collaboration ${ }^{(17)}$. Por se tratar de variáveis dicotômicas, foi utilizado o modelo de efeito fixo (odds ratio e intervalo de confiança de 95\%). Para a avaliação da heterogeneidade dos estudos, utilizou-se o teste de qui-quadrado de MantaelHaenzel e o teste de inconsistência de Higgins $\left(\mathrm{I}^{2}\right)$. Os estudos foram organizados por meio da elaboração de planilhas do Microsoft Excel 2013.

Foram identificados 1.341 estudos, pela busca abrangente nas bases de dados: 3 na Lilacs, 101 na Medlinel Pubmed, 96 na Scopus, 314 na CINAHL, 293 na Science Direct, 6 na Cochrane Library, 20 na Web of Science, 499 no Google Scholar e 9 na OpenGrey. Posteriormente, na análise do teste de relevância I, foram selecionados 37 artigos e pelo teste de relevância II, 26 estudos. A busca manual, por referência cruzada, resultou em seis artigos elegíveis e após leitura na íntegra, foi selecionado um estudo. Pela busca nas bases de dados e por referência cruzada, foram excluídos 127 artigos duplicados. A amostra final foi composta por 26 estudos. A caracterização dos 26 estudos selecionados pode ser visualizada na página a seguir e os mesmos estão dispostos de acordo com a ordem em que foram encontrados nas bases de dados. Os dados podem ser visualizados no Quadro 2, na página seguinte.

Quadro 2 - Caracterização dos estudos selecionados para a revisão sistemática e nível de evidência científica de acordo com o sistema GRADE.

\begin{tabular}{|c|c|c|c|c|c|}
\hline Autor & País/Ano & Método & Intervenção & Desfecho & GRADE \\
\hline Seratti et al ${ }^{(18)}$ & Itália 2016 & $\begin{array}{l}\text { Estudo documental } \\
\text { prospectivo }\end{array}$ & $\begin{array}{l}\text { Agachamento e posições } \\
\text { laterais } x \text { litotomia }\end{array}$ & $\begin{array}{l}\text { Maiores taxas de lacerações graves } \\
\text { em posição litotômica. }\end{array}$ & $2 \mathrm{C}$ \\
\hline Meyvis et al ${ }^{(19)}$ & $\begin{array}{l}\text { Bélgica } \\
2012\end{array}$ & $\begin{array}{c}\text { Estudo documental } \\
\text { transversal retrospectivo }\end{array}$ & Lateral $x$ litotomia & $\begin{array}{l}\text { Menores taxas de lacerações e } \\
\text { maiores de períneo íntegro na } \\
\text { adoção de posição lateral. }\end{array}$ & $1 \mathrm{C}$ \\
\hline Thies-Lagergren et al $\mathrm{al}^{(20)}$ & $\begin{array}{l}\text { Suécia } \\
2011\end{array}$ & $\begin{array}{c}\mathrm{ECR}^{*} \\
\text { controlado }\end{array}$ & $\begin{array}{l}\text { Banqueta } \times \text { litotomia e } \\
\text { outras posições horizontais }\end{array}$ & $\begin{array}{l}\text { Não houve aumento de } \\
\text { lacerações perineais não graves e } \\
\text { graves com o uso da banqueta. }\end{array}$ & $4 \mathrm{~A}$ \\
\hline Brément et $\mathrm{al}^{(21)}$ & $\begin{array}{l}\text { França } \\
2007\end{array}$ & $\mathrm{ECR}^{*}$ & $\begin{array}{l}\text { Decúbito lateral } \mathrm{x} \text { decúbito } \\
\text { dorsal }\end{array}$ & $\begin{array}{l}\text { Menores taxas de lacerações } \\
\text { perineais em decúbito lateral. }\end{array}$ & $4 \mathrm{~A}$ \\
\hline Hastings- Tolsma et al ${ }^{(22)}$ & $\begin{array}{l}\mathrm{EUA}^{+} \\
2007\end{array}$ & $\begin{array}{l}\text { Estudo documental } \\
\quad \text { retrospectivo }\end{array}$ & Lateral $x$ litotomia & $\begin{array}{l}\text { Maior risco de lacerações em } \\
\text { posição litotômica. }\end{array}$ & $1 \mathrm{C}$ \\
\hline $\begin{array}{l}\text { Nasir; Korejo; } \\
\text { Noorani }\end{array}$ & $\begin{array}{l}\text { Paquistão } \\
2007\end{array}$ & Estudo de caso controle & Agachamento $\mathrm{x}$ litotomia & $\begin{array}{c}\text { Redução das taxas de lacerações } \\
\text { perineais na posição de } \\
\text { agachamento. }\end{array}$ & $2 \mathrm{C}$ \\
\hline Ragnar et $\mathrm{al}^{(24)}$ & $\begin{array}{l}\text { Suécia } \\
2006\end{array}$ & $\mathrm{ECR}^{*}$ & 4 apoios versus sentada & $\begin{array}{l}\text { Posição de quatro apoios deve ser } \\
\text { encorajada pelos profissionais. }\end{array}$ & $4^{\mathrm{a}}$ \\
\hline Bodner-Adler et al $\left.\right|^{(25)}$ & $\begin{array}{l}\text { Áustria } \\
2003\end{array}$ & Estudo de caso-controle & Vertical $x$ supina & $\begin{array}{l}\text { Não houve associação entre } \\
\text { posição horizontal e ocorrência de } \\
\text { lacerações perineais. }\end{array}$ & $2 \mathrm{C}$ \\
\hline Bomfim-Hyppólito ${ }^{(26)}$ & $\begin{array}{l}\text { Brasil } \\
1998\end{array}$ & $\mathrm{ECR}^{*}$ & Vertical $\mathrm{x}$ horizontal & $\begin{array}{l}\text { Menor incidência de lacerações de } \\
2^{\circ} \text { grau em posição vertical. }\end{array}$ & $2 B$ \\
\hline
\end{tabular}


...continuação

\begin{tabular}{|c|c|c|c|c|c|}
\hline Autor & País/Ano & Método & Intervenção & Desfecho & GRADE \\
\hline De Jong et $\mathrm{al}^{(27)}$ & $\begin{array}{l}\text { África do } \\
\text { Sul } \\
1997\end{array}$ & $\mathrm{ECR}^{*}$ & $\begin{array}{l}\text { Posições verticais } x \\
\text { posições horizontais }\end{array}$ & $\begin{array}{c}\text { Maiores taxas de lacerações na } \\
\text { posição litotômica; } 3^{\circ} \text { grau foram } \\
\text { ligeiramente menores na posição } \\
\text { ereta. }\end{array}$ & $4 \mathrm{~A}$ \\
\hline Waldenstrom; Gottval(28) & $\begin{array}{l}\text { Suécia } \\
1996\end{array}$ & $\mathrm{ECR}^{*}$ & Banqueta $x$ supina & $\begin{array}{l}\text { Sem diferenças significativas entre } \\
\text { os dois grupos. }\end{array}$ & $3 B$ \\
\hline Golay; Vedam; Sorger ${ }^{(29)}$ & $\begin{array}{l}\mathrm{EUA}^{+} \\
1993\end{array}$ & Estudo de coorte & $\begin{array}{l}\text { Cócoras/agachamento x } \\
\text { semi sentada }\end{array}$ & $\begin{array}{l}\text { Agachamento deve ser encorajado } \\
\text { para prevenir lacerações perineais. }\end{array}$ & $1 \mathrm{C}$ \\
\hline $\begin{array}{l}\text { Gardosi; Sylvester; } \\
\text { B-Lynch }^{(30)}\end{array}$ & $\begin{array}{l}\text { Inglaterra } \\
1989\end{array}$ & $\mathrm{EC}^{\ddagger}$ controlado & $\begin{array}{l}\text { Cócoras, joelhos, sentada } \\
\text { na posição vertical ou em } \\
\text { pé x deitada }\end{array}$ & $\begin{array}{l}\text { Redução de lacerações graves na } \\
\text { posição cócoras. }\end{array}$ & $2 B$ \\
\hline $\begin{array}{l}\text { Gardosi; Hutson; } \\
\text { B-Lynch }\end{array}$ & $\begin{array}{l}\text { Reino } \\
\text { Unido } \\
1989\end{array}$ & $\begin{array}{c}\mathrm{ECR}^{*} \\
\text { controlado }\end{array}$ & Cócoras $\mathrm{x}$ reclinada & $\begin{array}{l}\text { Maior incidência de períneo } \\
\text { íntegro e menor de lacerações de } \\
2^{\circ} \text { grau, na posição de cócoras. }\end{array}$ & $2 \mathrm{~B}$ \\
\hline Stewart;Hillan; Calder ${ }^{(32)}$ & $\begin{array}{l}\text { Escócia } \\
1983\end{array}$ & $\mathrm{ECR}^{*}$ & $\begin{array}{l}\text { Banqueta (cócoras) x } \\
\text { decúbito dorsal }\end{array}$ & $\begin{array}{l}\text { Banqueta pode ser utilizada para } \\
\text { preservação perineal. }\end{array}$ & $3 B$ \\
\hline $\begin{array}{l}\text { Gottvall; Allebeck; } \\
\text { Ekéus }^{(33)}\end{array}$ & $\begin{array}{l}\text { Suécia } \\
2007\end{array}$ & Estudo de coorte & $\begin{array}{c}\text { Posições verticalizas } \\
\text { (banqueta, semi sentada } \\
\text { lateral encostada, } \\
\text { agachada, } 4 \text { apoios, em pé) } \\
\text { x litotomia e supina }\end{array}$ & $\begin{array}{l}\text { Litotomia e agachamento } \\
\text { podem aumentar lacerações } \\
\text { graves. Efeitos globais de várias } \\
\text { posições são inconclusivos. }\end{array}$ & $2^{\mathrm{a}}$ \\
\hline Da Silva et $\mathrm{al}^{(34)}$ & $\begin{array}{l}\text { Brasil } \\
2012\end{array}$ & $\begin{array}{l}\text { Estudo transversal } \\
\text { retrospectivo }\end{array}$ & $\begin{array}{l}\text { Posições verticalizas x } \\
\text { decúbito dorsal }\end{array}$ & $\begin{array}{l}\text { Posições alternativas à dorsal, } \\
\text { podem melhorar a integridade } \\
\text { perineal. }\end{array}$ & $2 \mathrm{~B}$ \\
\hline Terry et $\mathrm{al}^{(35)}$ & $\begin{array}{l}\mathrm{EUA}^{+} \\
2006\end{array}$ & $\mathrm{EC}^{\ddagger}$ não randomizado & $\begin{array}{l}\text { Sentada/agachada/4 apoios } \\
\text { x supina }\end{array}$ & $\begin{array}{l}\text { Posições verticais podem reduzir } \\
\text { lacerações perineais. }\end{array}$ & $2 \mathrm{~B}$ \\
\hline Albers et $\mathrm{al}^{(36)}$ & $\begin{array}{l}\text { México e } \\
\mathrm{EUA}^{+} \\
1996\end{array}$ & Estudo de coorte & $\begin{array}{l}\text { Semi-sentada, sentada, } \\
\text { lateral, outras posições x } \\
\text { litotomia }\end{array}$ & $\begin{array}{c}\text { Posição litotômica não deve ser } \\
\text { utilizada para prevenir lacerações } \\
\text { perineais. }\end{array}$ & $1 \mathrm{C}$ \\
\hline Maheux-Lacroix et $\mathrm{al}^{(37)}$ & $\begin{array}{l}\text { Canadá } \\
2013\end{array}$ & $\begin{array}{l}\text { Estudo exploratório } \\
\text { retrospectivo comparativo }\end{array}$ & $\begin{array}{l}\text { Posições verticalizadas x } \\
\text { decúbito dorsal }\end{array}$ & $\begin{array}{c}\text { Posições verticalizadas podem } \\
\text { estar associadas a menores taxas } \\
\text { de lacerações vaginais. }\end{array}$ & $1 \mathrm{C}$ \\
\hline Roberts; Kriz ${ }^{(38)}$ & $\begin{array}{l}\mathrm{EUA}^{+} \\
1984\end{array}$ & $\begin{array}{l}\text { Estudo descritivo } \\
\text { correlacional retrospectivo }\end{array}$ & $\begin{array}{l}\text { Semi-sentada/4 apoios/sims } \\
\text { lateral/agachamento/em pé } \\
\text { x litotomia }\end{array}$ & $\begin{array}{l}\text { Não foi possível concluir que a } \\
\text { posição influencie o resultado } \\
\text { perineal. }\end{array}$ & $1 \mathrm{C}$ \\
\hline $\begin{array}{l}\text { Gupta; Brayshaw; } \\
\text { Lilford }^{(39)}\end{array}$ & $\begin{array}{l}\text { Reino } \\
\text { Unido } \\
1989\end{array}$ & $\mathrm{ECR}^{*}$ & Agachamento x litotomia & $\begin{array}{l}\text { Lacerações de } 2^{\circ} \text { grau foram } \\
\text { mais comuns na posição de } \\
\text { agachamento. }\end{array}$ & $3 \mathrm{~A}$ \\
\hline $\begin{array}{l}\text { Schirmer; Fustinoi; } \\
\text { Basile }^{(40)}\end{array}$ & $\begin{array}{l}\text { Brasil } \\
2011\end{array}$ & $\mathrm{ECR}^{*}$ & $\begin{array}{l}\text { Lateral esquerda } x \text { ereta } \\
\text { semi-sentada }\end{array}$ & $\begin{array}{l}\text { Maior frequência de lacerações de } \\
1^{\circ} \text { grau em posição lateral. }\end{array}$ & $4 \mathrm{~A}$ \\
\hline Altman et $\mathrm{al}^{(41)}$ & $\begin{array}{l}\text { Suécia } \\
2007\end{array}$ & $\mathrm{ECR}^{*}$ & 4 apoios $x$ sentada & $\begin{array}{l}\text { Quatro apoios e sentada na } \\
\text { posição vertical, não apresentam } \\
\text { diferenças significativas nas } \\
\text { lacerações graves. }\end{array}$ & $4 \mathrm{~A}$ \\
\hline Baracho et $\mathrm{al}^{(42)}$ & $\begin{array}{l}\text { Brasil } \\
2009\end{array}$ & Estudo de coorte & $\begin{array}{l}\text { Cócoras/sentada/semi- } \\
\text { sentada x decúbito dorsal/ } \\
\text { litotomia }\end{array}$ & $\begin{array}{l}\text { Sem associação entre posição } \\
\text { horizontal e ocorrência de } \\
\text { lacerações perineais. }\end{array}$ & $2 \mathrm{~A}$ \\
\hline Heinz et $\mathrm{al}^{(43)}$ & $\begin{array}{l}\text { Áustria } \\
2001\end{array}$ & Estudo de caso-controle & Banqueta x posição dorsal & $\begin{array}{l}\text { Banqueta pode ser uma alternativa } \\
\text { no parto. }\end{array}$ & $2 \mathrm{C}$ \\
\hline
\end{tabular}

* Estudo Clinico Randomizado

${ }^{+}$Estados Unidos da América

‡Estudo Clínico

Foi realizada uma avaliação estatística dos estudos metodologicamente semelhantes (estudos clínicos randomizados), denominada de metanálise, e, posteriormente a síntese dos resultados obtidos. A amostra final comtemplou oito artigos.
Para o desfecho principal do estudo, a prevenção de lacerações perineais e consequente ocorrência de períneo íntegro, foram selecionados oito estudos para a realização da metanálise, por apresentaram a adoção de posições verticalizadas no parto comparada a posições horizontais (Figura 2). 


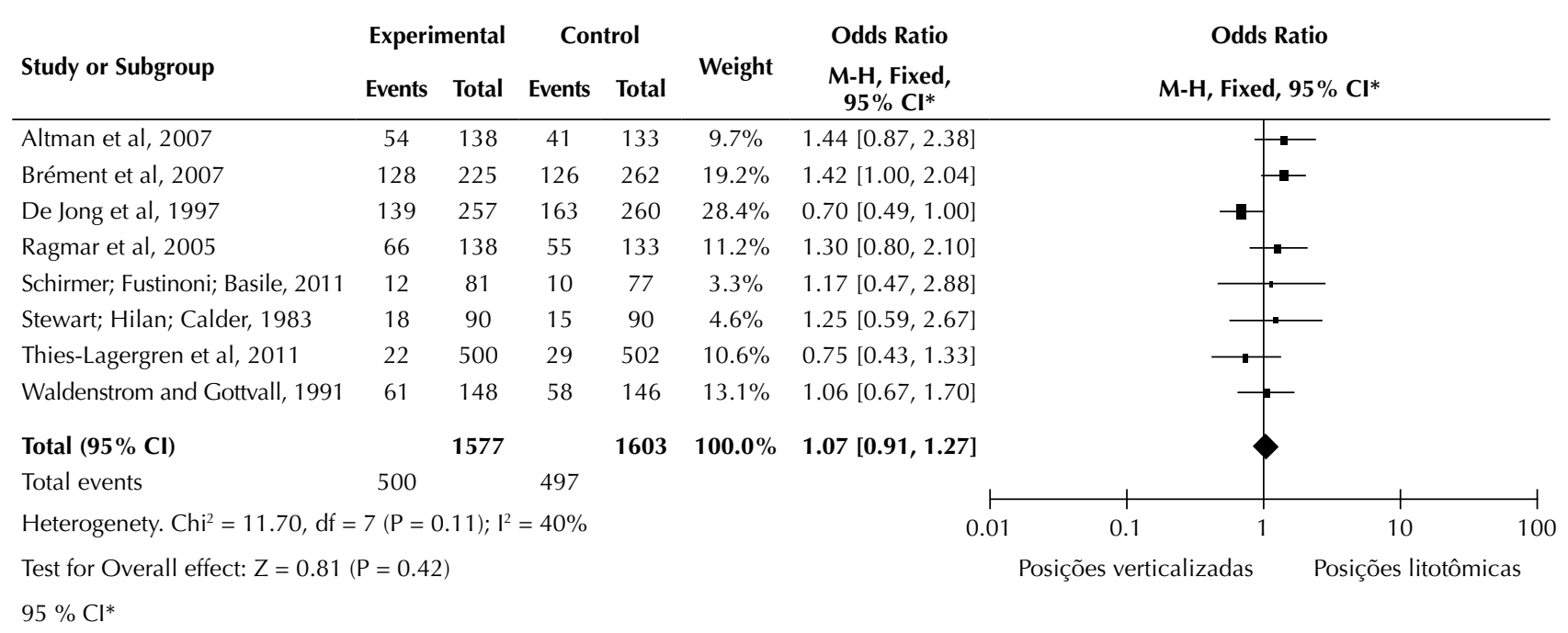

* Control Interval

Figura 2 - Metanálise de posições verticalizadas versus posições horizontais para períneo íntegro e gráfico de funnel plot, elaborado pela ferramenta RevMan.

Em quatro estudos, os autores compararam a posição de cócoras no parto, com posições litotômicas. Na primeira publicação, 500 mulheres adotaram a posição de cócoras (grupo experimental), e destas, 22 (44\%) apresentaram períneo íntegro, comparadas com 29 (17\%) das que adotaram posições litotômicas ${ }^{(20)}$.

$\mathrm{Na}$ segunda publicação elencada, a diferença estatística não foi significativa, pois, de 90 mulheres que tiveram parto de cócoras na banqueta, 18 (5\%) tiveram períneo íntegro versus $15(6 \%)$ das que adotaram posições horizontais ${ }^{(32)}$. O mesmo ocorre em estudo mais antigo, publicado em 1991, pois, das 148 mulheres que tiveram parto de cócoras, 27 $(5,4 \%)$ tiveram períneo íntegro, comparado com $22(6,6 \%)$ de 146 das mulheres em posição supina ${ }^{(28)}$.

Outras duas publicações compararam posição de quatro apoios com a posição sentada. Na primeira, apenas 2\% (66 mulheres de 138) apresentaram períneo íntegro em quatro apoios, contra 55 mulheres $(2,4)$ de 133 que adotaram posição sentada ${ }^{(24)}$. Já, na segunda publicação, 54 mulheres de 138 (51\%) tiveram esse desfecho adotando a posição de quatro apoios e $41(37 \%)$ de 133 na posição sentada.

Dois estudos, respectivamente de 2007 e 2011, analisaram o efeito da posição lateral versus decúbito dorsal e semi-sentada, respectivamente, onde 128 mulheres em posição lateral apresentaram períneo íntegro $(56,9 \%)$ versus $48,1 \%$ em decúbito dorsal ${ }^{(21)}$. No segundo estudo, 12 mulheres $(14,8 \%)$ de 81 tiveram períneo íntegro em posição lateral e $10(13 \%)$ em posição semi-sentada ${ }^{(40)}$.

Em pesquisa mais abrangente, o qual comparou diversas posições verticais versus horizontais, 139 (2\%) de 257 mulheres tiveram períneo íntegro na posição vertical e 163 $(1,5 \%)$ de 260 na posição horizontal( ${ }^{(27)}$.
Por meio do aplicativo Review Manager (RevMan), foi desenvolvido o gráfico da metanálise dos estudos clínicos randomizados selecionados, também denominado de funnel plot ou gráfico de floresta, o qual encontra-se na Figura 2.

De acordo com o teste do $\mathrm{I}^{2}$, a heterogeneidade dos estudos pode ser classificada como moderada. Pode-se inferir que alguns fatores de confusão podem ter ocasionado a heterogeneidade. Identificou-se que não existe diferença estatística significativa entre os grupos experimental e controle $(\mathrm{p}=0,90$; OR 0,99; IC 95\% 0,82-1,20).

Em relação aos estudos que não foram incluídos na metanálise, apenas seis analisaram o desfecho períneo íntegro. Os demais estudos analisaram somente o desfecho lacerações perineais não graves e graves, e foram selecionados para esta pesquisa, com o objetivo de subsidiar outros artigos que poderão ser publicados. De acordo com os dados apresentados por esses estudos, a maioria demonstrou aumento da ocorrência de períneo íntegro em mulheres que adotaram posições verticalizadas no parto. Os estudos que apresentaram o desfecho lacerações perineais apontaram menores taxas de traumas perineais em mulheres que adotaram posições verticalizas, principalmente lacerações graves. Já, em partos que ocorreram na banqueta, a maioria dos estudos apresentou taxas elevadas de lacerações graves ( $3^{\circ}$ e $4^{\circ}$ graus).

De acordo com o Sistema GRADE, a qualidade da evidência científica dos estudos clínicos randomizados, incluídos na metanálise, foi considerada alta, a favor de uma ação, apesar da grande semelhança estatística dos resultados dos estudos não apontar para um resultado totalmente a favor de uma posição ou outra (Quadro 3). 
Quadro 3 - Sumário de resultados da qualidade da evidência científica de acordo com o Sistema GRADE, elaborado pelo aplicativo Guideline Development Tool.

Sumário de Resultados

Posições verticalizadas comparado a posições litotômicas para períneo íntegro

Paciente ou população: parturientes

Contexto: trabalho de parto normal

Intervenção: posições verticalizadas

Comparação: posições litotômicas

\begin{tabular}{|c|c|c|c|c|c|c|}
\hline \multirow{2}{*}{ Desfechos } & \multicolumn{2}{|c|}{ Efeitos absolutos potenciais $\left(95 \%\right.$ IC $\left.{ }^{\dagger}\right)$} & \multirow{2}{*}{$\begin{array}{l}\text { Efeito relativo } \\
\quad(95 \% \mathrm{IC})\end{array}$} & \multirow{2}{*}{$\begin{array}{c}\mathrm{N}^{\circ} \text { de participantes } \\
\text { (estudos) }\end{array}$} & \multirow{2}{*}{$\begin{array}{l}\text { Qualidade da } \\
\text { evidência } \\
\left.\text { (GRADE }{ }^{*}\right)\end{array}$} & \multirow{2}{*}{ Comentários } \\
\hline & $\begin{array}{l}\text { Risco com posições } \\
\text { litotômicas }\end{array}$ & $\begin{array}{l}\text { Risco com Posições } \\
\text { verticalizadas }\end{array}$ & & & & \\
\hline $\begin{array}{l}\text { Desfecho } \\
\text { períneo íntegro }\end{array}$ & 310 por 1.000 & $\begin{array}{l}322 \text { por } 1.000 \\
\text { (295 para } 353)\end{array}$ & $\begin{array}{c}\mathrm{RR}^{\S} \\
1.04 \\
(0.95 \text { para } 1.14)\end{array}$ & $\begin{array}{c}3083 \\
\left(8 \mathrm{ECRs}^{*}\right)\end{array}$ & 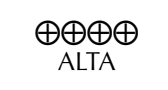 & \\
\hline
\end{tabular}

Os níveis de qualidade do grupo de trabalho do GRADE ${ }^{\dagger}$

Qualidade Alta: Existe muita confiança que o efeito real encontra-se próximo ao efeito estimado

Qualidade Moderada: Existe moderada confiança no efeito estimado: O efeito real está provavelmente próximo ao efeito estimado, mas há

possibilidade que seja substancialmente diferente

Qualidade Baixa: A confiança no efeito estimado é limitada: o efeito real pode ser substancialmente diferente da estimativa de efeito

Qualidade Muito Baixa: Existe muito pouca confiança no efeito estimado: O efeito real é provavelmente substancialmente diferente do efeito estimado

${ }^{+}$Intervalo de Confiança

${ }^{\ddagger}$ Grading of Recommendations Assessment, Development and Evaluation

$\S$ Risco Relativo

* Estudos Clínicos Randomizados

\section{DISCUSSÃO}

As posições verticalizadas no parto cada vez mais tem sido alvo dos estudos na área obstétrica, por constituírem uma das boas práticas de atenção ao parto e nascimento, que contribui para a humanização da assistência e para o protagonismo da mulher no trabalho de parto e parto.

A principal justificativa para apoiar a adoção de posições verticalizadas no parto é a ação gravitacional, a qual contribui na descida do feto pelo canal vaginal, além de modificar a angulação da pelve materna. Já, na posição litotômica, o canal vaginal apresenta uma curvatura para cima, dificultando a descida fetal no período expulsivo ${ }^{(44)}$.

A partir disso, muitos estudos tem por objetivo analisar riscos e benefícios das posições verticalizas no parto, comparadas às posições horizontais. No entanto, percebe-se que, em sua maioria, são pesquisas desatualizadas e que não analisaram o desfecho períneo íntegro, foco principal desta metanálise, apenas lacerações perineais. Além disso, nem todos os resultados apresentam diferenças significativas entre as posições ou defendem que a posição supina seria a melhor escolha para as parturientes.

Em uma metanálise de 20 estudos, sobre posições no período expulsivo do parto em mulheres com analgesia peridural, não se chegou a uma conclusão precisa sobre eficácia das posições verticalizadas no parto. Porém, a análise estatística demonstrou aumento de lacerações de $2^{\circ}$ grau em mulheres que deram à luz em banqueta ${ }^{(45)}$.

Em estudo de coorte italiano que comparou diferentes posições de parto com variáveis maternas e neonatais, em
$100 \%$ das mulheres que adotaram a posição litotômica, foi realizada episiotomia. Esse é o principal fator confundidor para os dados, dificultando uma comparação fidedigna. Salienta-se que o número de lacerações em posições verticalizadas, nesse estudo, foi estatisticamente maior. Também pode-se inferir que a posição litotômica contribui para o aumento de intervenções como a episiotomia. Além disso, esse estudo conclui que existem muitos benefícios na adoção de posições verticais, durante o processo de trabalho de parto normal, principalmente na rotação de bebês em variedade de posição occipto posterior para occipto anterior ${ }^{(46)}$.

Em revisão sistemática publicada recentemente, foram selecionados 22 estudos clínicos, os quais objetivaram avaliar as posições verticalizadas no segundo estágio de trabalho de parto e sua importância no desfecho materno-fetal. Em relação ao períneo feminino, os estudos demonstram que as posições verticalizadas foram eficazes na redução significativa da prática da episiotomia. Enquanto alguns estudos apresentaram que a integridade perineal é mais preservada quando a mulher adota uma posição vertical, outros estudos demonstraram o oposto. Essa heterogeneidade significativa entre os estudos, impossibilitou que se chegasse a uma conclusão definitiva, porém os benefícios da posição verticalizada são maiores que os riscos e a mesma deve ser encorajada pelos profissionais, respeitando a preferência da mulher ${ }^{(47)}$.

Corroborando com algumas considerações desse mesmo estudo, pontua-se que, na presente metanálise, foram identificados fatores que podem influenciar negativamente nos resultados. Por exemplo, a falta de controle sobre o tempo de permanência das mulheres em posições verticalizadas, 
bem como, a adoção dessas posições pelas mesmas, pois, muitas não conseguem se manter nessa posição. Também é importante ressaltar que existem outros fatores que podem influenciar na ocorrência de lacerações e na integridade perineal e que não foram controlados pelo estudo, tais como, o uso de técnicas para proteção perineal e redução de lacerações, as condições do períneo, a prática da episiotomia (realizada na maioria dos partos em posição litotômica e que acabam por confundir os resultados), a condução dos puxos pelo profissional de saúde, a paridade das mulheres, dentre outros aspectos.

Reitera-se que, nesta metanálise, as posições verticalizadas foram incluídas, porém, alguns estudos trazem comparações de cada tipo de posição vertical (quatro apoios, agachamento, lateral) e posição supina (semi-sentada, decúbito dorsal), o que também pode influenciar nos resultados.

Pontua-se que, ocorreram limitações neste estudo, devido às metodologias utilizadas pelos artigos elencados, no que se refere ao controle de diversas variáveis que podem interferir no desfecho períneo íntegro. Também, por terem sido elencados estudos que contemplassem parto vaginal de baixo risco, a maioria dessas variáveis não foi considerada, tais como, indução de trabalho de parto e analgesia. Sugere-se mais estudos sobre a temática, a partir de estudos com metodologias mais controladas.

\section{CONCLUSÃO}

Por meio da metanálise dos estudos, não foi possível afirmar que as posições verticalizadas previnem lacerações perineais, em comparação com as posições horizontais, pois, não houve diferença estatística significativa.
Porém, a partir dos resultados, foram identificadas as seguintes evidências: apesar da pequena diferença estatística, a adoção de posições verticalizadas, no parto normal, deve ser encorajada pelos profissionais, pois pode evitar traumas graves com necessidade de sutura, e, pode contribuir para a integridade perineal. A posição litotômica deve ser utilizada com cautela pelos profissionais, pois, pode aumentar o risco de lacerações perineais graves e intervenções obstétricas desnecessárias, tais como a episiotomia.

O estudo aponta que não é possível afirmar com precisão a eficácia das posições verticalizadas em detrimento das posições horizontais para o desfecho períneo íntegro, mas, a livre escolha da mulher pela posição no parto deve ser encorajada, principalmente, e a mesma deve ser orientada quanto a riscos e benefícios de posições verticalizadas e horizontais.

Por fim, é premente afirmar a importância do papel das enfermeiras obstétricas e obstetrizes na mudança de paradigma do modelo obstétrico, visto que, cada vez mais o setor saúde oportuniza uma formação diferenciada e ampliada. O conhecimento dessas profissionais considera as evidências científicas como suporte para o cuidado e a tomada de decisão, possibilitando o protagonismo do parto à mulher, por meio do respeito aos seus direitos previstos em lei e pelo viés da humanização da assistência ao parto e nascimento. Para além do conhecimento científico, essas profissionais também possuem a sensibilidade de entender o processo de parto como um momento único e subjetivo da mulher, que deve respeitar a fisiologia do corpo feminino e utilização as práticas invasivas quando estritamente necessário.

\section{RESUMO}

Objetivo: Investigar se a adoção de posições verticalizadas pela mulher, no parto, comparada à posição litotômica, previne lacerações perineais. Método: Revisão sistemática com metanálise. As buscas foram realizada nas bases de dados: LILACS, Medline/PubMed, CINAHL, Cochrane Library, Web of Science, Science Direct e Scopus. As buscas na literatura cinzenta foram conduzidas nas bases Google Scholar e OpenGrey. Também foram consideradas as listas de referências dos artigos incluídos. Para análise da qualidade metodológica dos artigos, utilizou-se a ferramenta da colaboração Cochrane e a ACROBAT-NRSI. Resultados: Foram elencados 26 estudos e 8 foram selecionados para a metanálise. O nível de evidência científica foi classificado pelo Sistema GRADE e considerado alto. Não houve diferença estatística significativa entre posições verticalizadas em relação as posições horizontais. Apesar dessa constatação, as posições verticalizadas apresentaram índices reduzidos de lacerações perineais graves. Conclusão: A adoção de posições verticalizadas, no parto normal, pode ser encorajada pelos profissionais, pois pode evitar lacerações perineais graves, porém, não é possível afirmar com precisão a eficácia destas em detrimento das posições horizontais para o desfecho períneo íntegro.

\section{DESCRITORES}

Enfermagem Obstétrica; Parto; Modalidades de Posição; Lacerações; Revisão; Metanálise.

\section{RESUMEN}

Objetivo: Investigar si la adopción de posiciones verticales por parte de la mujer en el parto, en comparación con la posición de litotomía, previene las laceraciones perineales. Método: Revisión sistemática con metaanálisis. Se realizaron búsquedas en las siguientes bases de datos: LILACS, Medline/PubMed, CINAHL, Cochrane Library, Web of Science, Science Direct y Scopus. Se realizaron búsquedas en la literatura gris en las bases de datos Google Scholar y Opengray. También se examinaron las listas de referencia de los artículos incluidos. La herramienta de colaboración Cochrane y el ACROBAT-NRSI se utilizaron para analizar la calidad metodológica de los artículos. Resultados: Se enumeraron 26 estudios y se seleccionaron 8 para el metaanálisis. El nivel de evidencia científica fue clasificado por el Sistema GRADE y considerado alto. No hubo una diferencia estadística significativa entre las posiciones verticales y las horizontales. A pesar de este hallazgo, las posiciones verticales presentaban bajas tasas de laceraciones perineales graves. Conclusión: La adopción de posiciones verticales en el parto normal puede ser fomentada por los profesionales, ya que puede evitar desgarros perineales severos, sin embargo, no es posible afirmar con exactitud su eficacia en detrimento de las posiciones horizontales para el resultado perineal completo.

\section{DESCRIPTORES}

Enfermería Obstetrica; Parto; Modalidades de Posición; Laceraciones; Revisión; Metaanálisis. 


\section{REFERÊNCIAS}

1. Souza JP, Pileggi-Castro C. On labor and childbirth: the importance of quartenary prevention. Cad Saúde Pública. 2014;30 Suppl 1:S11-13. DOI: http://dx.doi.org/10.1590/0102-311XPE02S114

2. Leal MC, Pereira APE, Domingues RMSM, Filha MMT, Dias MAB, Pereira MN, et al. Obstetric interventions during labor and childbirth in Brazilian Low-risk women. Cad Saúde Pública. 2014;30 Suppl 1:S17-32. DOI: http://dx.doi.org/10.1590/0102-311X00151513

3. Andreucci CB, Cecatti JG. Desempenho de indicadores de processo do Programa de Humanização do Pré-Natal e Nascimento no Brasil: uma revisão sistemática. Cad Saúde Pública. 2011;27(6):1053-164. DOI: http://dx.doi.org/10.1590/S0102-311X2011000600003

4. Ramalho KS, Da Silva ST, De Lima SM, Santos MA. Política de saúde da mulher à integralidade: efetividade ou possibilidade? Cad Grad Cienc Hum Soc [Internet]. 2012 [citado 2017 ago. 10]. 1(1):11-22. Disponível em: https://periodicos.set.edu.br/index.php/fitshumanas/ article/view/462/198

5. Oliveira FAM, Leal GCG, Wolff LDG, Rabelo M, Poliquesi CB. Refletions on the nurse's role in the Rede Cegonha (stork network). Rev Enferm UFPE. 2016;10(2):867-74. DOI: 10.5205/reuol.6884-59404-2-SM-1.1002sup201622

6. World Health Organization. WHO recommendations: intrapartum care for a positive childbirth experience [Internet]. Geneva: WHO; 2018 [cited 2019 Mar 25]. Available from: https://apps.who.int/iris/bitstream/handle/10665/260178/9789241550215-eng. pdf?sequence $=1$

7. España. Ministerio de Ciencia y Innovación. Clinical practice guideline on care in normal birth [Internet]. Galicia: PAH; 2013 [cited 2016 Apr 19]. Available from: https://portal.guiasalud.es/wp-content/uploads/2018/12/GPC_472_Parto_Normal_Osteba_compl_en.pdf

8. Brasil. Ministério da Saúde. Diretrizes Nacionais de Assistência Ao Parto Normal: versão resumida [Internet]. Brasília; 2016 [citado 2016 fev. 10]. Disponível em: http://conitec.gov.br/images/Protocolos/Diretrizes/Diretrizes_PartoNormal_VersaoReduzida_FINAL.pdf

9. Côrtes CT, Oliveira SMJV, Santos RCS, Francisco AA, Riesco MLG, Shimoda GT. Implementation of evidence-based practices in normal delivery care. Rev Latino Am Enfermagem. 2018;26:e2988. DOI: http://dx.doi.org/10.1590/1518-8345.2177.2988

10. Silva LS, Leão DCMR, Cruz AFN, Alves VH, Rodrigues DP, Pinto CB. Women's knowledge about the diferente positions for labour: a contribuition for caring. Rev Enferm UFPE. 2016;10 (4):3531-6. DOI: 0.5205/reuol.9681-89824-1-ED.1004sup201604

11. Higgins JPT, Green S, editors. Cochrane Handbook for Systematic Reviews Of Interventions. Version 5.0.2. [Internet]. 2009 [cited 2016 Apr 12]. Available from: https://training.cochrane.org/handbook

12. National Health and Medical Research Council (NHMRC). A guide to the development, implementation and evaluation of clinical practice guidelines [Internet]. Canberra; 1999 [cited 2016 Feb 10]. Available from: http://www.nhmrc.gov. au/publications/synopses/ cp30syn.htm

13. Moher D, Liberati A, Tetzlaff J, Altman DG. Preferred reporting items for systematic reviews and meta-analyses: the PRISMA Statement. Epidemiol Serv Saúde. 2015;24(2):335-42. DOI: 10.1371/journal.pmed.1000097

14. Higgins JPT, Altman DG, Gotzsche PC, Jüni P, Moher D, Oxman AD, et al. The Cochrane Collaboration's tool for assessing risk of bias in randomised trials. Br Med J. 2011;343(d5928):889-93. DOI: 10.1136/bmj.d5928

15. Sterne J, Higgins J, Reeves B, editors. Cochrane Methds. Risk of bias assessment tool: for non-randomized studies of interventions (ACROBAT-NRSI). Version 1.0.0. [Internet]. 2014 Available from: https://methods.cochrane.org/news/risk-bias-assessment-tool-nonrandomised-studies-interventions

16. GRADE Working Group. The Grading of recommendations assessment development and evaluation [Internet]. Chicago; 2014 [cited 2017 Dec 20]. Available from: http://www.gradeworkinggroup.org

17. Higgins JPT, Green S, editors. Cochrane Handbook for Systematic Reviews of Interventions 4.2.6. [Internet]. 2006 [cited 2016 Apr 12]. Available from: http://www.cochrane.org/resources/handbook/hbook.htm

18. Seratti M, Di Dedda MC, Bogani G, Sorice P, Cromi A, Uccella S, et al. Position in the second stage of labour and de novo onset of post-partum urinary incontinence. Int Urogynecol J Pelvic Floor Dysfunct. 2016;27(2):281-6. DOI: 10.1007/s00192-015-2829-z

19. Meyvis I, Rompaey BV, Goormans K, Truijen S, Lambers S, Mestdagh E, et al. Maternal position and other variables: effects on perineal outcomes in 557 birth. Birth. 2012;39(2):1-6. DOI: 10.1111/j.1523-536X.2012.00529.x

20. Thies-Lagergren L, Kvist LJ, Christensson K, Hildingsson I. No reduction in instrumental vaginal births and no increased risk for adverse perineal outcome in nulliparous women giving birth on a birth seat: results of a Swedish randomized controlled trial. BMC Pregnancy Childbirth. 2011;11(22):1-9. DOOI: https://doi.org/10.1186/1471-2393-11-22

21. Brément S, Mossan S, Belery A, Racinet C. Accouchement en décubitus latéral. Essai clinique randomisé comparant les positions maternelles en décubitus lateral et en décubitus dorsal lors de la deuxième phase du travail. Gynecol Obstet Fertil. 2007;35(7-8):637-44. DOI: https://doi.org/10.1016/j.gyobfe.2007.04.022

22. Hastings- Tolsma M, Vincet D, Emeis C, Francisco T. Getting through birth in one piece. Am J Matern Child Nurs. 2007;32(3):158-64. DOI: 10.1097/01.NMC.0000269565.20111.92

23. Nasir A, Korejo R, Noorani KJ. Childbirth in squatting position. J Pak Med Assoc. 2007;57(1):19-22.

24. Ragnar I, Altman D, Tydén T, Olsson S-E. Comparison of the maternal experience and duration of labour in two upright delivery positions: a randomised controlled trial. BJOG. 2006; 113(2):165-70. DOI:10.1111/j.1471-0528.2005.00824.x

25. Bodner-Adler B, Bodner K, Kimberger O, Lozanov P, Husslein P, Mayerhofer K. Women's position during labour: influence on maternal and neonatal outcome. Wien Klin Wochenschr. 2003;115(19-20):720-23.

26. Bomfim-Hyppólito SB. Influence of the position of the mother at delivery over some maternal and neonatal outcomes. Int J Gynaecol Obstet. 1998;63 Suppl 1:S67-73.

27. De Jong PR, Johanson RB, Baxen P, Adrians VD, van der Westhuisen S, Jones PW. Randomised trial comparing the upright and supine positions for the second stage of labour. Br J Obstet Gynaecol.1997;104(5):567-71. 
28. Waldenstrom U, Gottvall K. A randomized trial of birthing stool or conventional semirecumbent position. Birth. 1991;18(1):5-10. DOI: 10.1111/j.1523-536X.1991.tb00045.x

29. Golay A, Vedam S, Sorger L. The squatting position for the second stage oflabor: effects on labor and on maternal and fetal well-being. Birth. 1993;20(2):73-8.

30. Gardosi J, Sylvester S, B-Lynch C. Alternative positions in the second stage of labour: a randomized controlled trial. Br J Obstet Gynaecol. 1989;96:1290-96. DOI: 10.1111/j.1523-536X.1993.tb00420.x

31. Gardosi J, Hutson N, B-Lynch C. Randomised controlled trial of squatting in the second stage of labour. Lancet. 1989;2(8654)74-77. DOI: http://dx.doi.org/10.1016/S0140-6736(89)90315-2

32. Stewart P, Hillan E, Calder AA. A randomised trial to evaluate the use of a birth chair for delivery. Lancet. 1983;1(8337):1296-8. DOI: http://dx.doi.org/10.1016/S0140-6736(83)92412-1

33. Gottvall K, Allebeck P, Ekéus C. Risk factors for anal sphincter tears: the importance of maternal position at birth. BJOG. 2007;114(10):1266-72. DOI:10.1111/j.1471-0528.2007.01482.x

34. Silva FMB, Oliveira SMJV, Bick D, Osava RH, Tuesta EF, Riesco MLG. Risk factors for birth-related perineal trauma: a cross-sectional study in a birth centre. J Clin Nurs. 2012;21(15-16):2209-18. DOI: 10.1111/j.1365-2702.2012.04133.x

35. Terry RR, Westcott J, O'Shea L, Kelly F. Postpartum outcomes in supine delivery by physicians vs nonsupine delivery by midwives. J Am Osteopath Assoc. 2006;106(4):199-202.

36. Albers LL, Anderson D, Cragin L, Daniels SM, Hunter C, Sedler KD, et al. Factors related to perineal trauma in childbirth. J Nurse Midwifery. 1996;41(4):269-74.

37. Maheux-Lacroix S, Tremblay M, Dubois N, Turcotte S, Girard N, Houde M et al. A new method of positioning at delivery compared with the dorsal recumbent position: an exploratory retrospective study of obstetric outcomes. J Obstet Gynaecol Can. 2013;35(6):523-30. DOI:10.1016/S1701-2163(15)30910-5

38. Roberts JE, Kriz DM. Delivery positions and perineal outcome. J Nurse Midwifery. 1984;29(3):186-90. DOI: 10.1016/0091-2182(84)90202-7

39. Gupta JK, Brayshaw EM, Lilford RJ. An experiment of squatting birth. Eur J Obstet Gynecol Reprod Biol. 1989;30:217-20.

40. Schirmer J, Fustinoni SM, Basile ALO. Perineal outcomes on the left lateral versus vertical semisitting birth positions: a randomized study. Acta Paul Enferm. 2011;24(6):745-50. DOI: http://dx.doi.org/10.1590/S0103-21002011000600002

41. Altman D, Ragnar I, Ekström A, Tydén T, Olsson S-E. Anal sphincter lacerations and upright delivery postures - a risk analysis from a randomized controlled trial. Int Urogynecol J Pelvic Floor Dysfunct. 2007;18(2):141-6. DOI: http://dx.doi.org/10.1007/s00192-006-0123-9.

42. Baracho SM, Figueiredo EM, Silva LB, Cangussu ICAG, Pinto DN, Souza ELBL, et al. The influence of vaginal birth position on obstetric and neonatal variables in primiparous women. Rev Bras Saúde Mater Infant. 2009;9(4):409-14. DOI: http://dx.doi.org/10.1590/S151938292009000400004

43. Heinz SS, Benedicic C, Arikan MG, Haas J, Petru E. Spontane vaginal geburt unter verwendung eines gebärhockers: vergleich mit der konventionellen rückenlage. Wien Klin Wochenshr. 2001;113:117-8.

44. Racinet C. Positions maternelles pour I'accouchement. Gynecol Obstet Fertil. 2005;33:533-38. DOI: 10.1016/S1297-9589(05)00190-6.

45. Gupta JK, Hofmeyr GJ, Smyth R. Position in the second stage of labour for women without epidural anaesthesia. Cochrane Database Syst Rev. 2012;(5):CD002006. DOI: 10.1002/14651858

46. Gizzo S, Di Gangi S, Noventa M, Bacile V, Zanbon A, Nardelli GB. Women's choice of positions during Labour: return to the past or a modern way to give birth? A cohort study in Italy. Biomed Res Int. 2014;2014:638093. DOI: http://dx.doi.org/10.1155/2014/638093.

47. Deliktas A, Kukulu K. A meta-analysis of the effect on maternal health of upright positions during the second stage of labour, without routine epidural analgesia. J Adv Nurs. 2018;74(2):263-78. DOI: 10.1111/jan.13447 